\title{
Mean-Variance Portfolio Allocation \\ with a Value at Risk Constraint
}

By

Enrique Sentana

DISCUSSION PAPER 380

May 2001

\section{FINANCIAL MARKETS GROUP}

AN ESRC RESEARCH CENTRE

\section{LONDON SCHOOL OF ECONOMICS}

Any opinions expressed are those of the author and not necessarily those of the Financial Markets Group. 


\title{
Mean-Variance Portfolio Allocation with a Value at Risk Constraint ${ }^{1}$
}

\author{
Enrique Sentana \\ (CEMFI, CEPR and LSE FMG)
}

January 2001

Revised: May 2001

\begin{abstract}
${ }^{1} D$ suhylrxv gudiwri vklv sdshu z dv fluf xolwhy xqghu wkh wweh Y Yoxh dwUlvn dqg

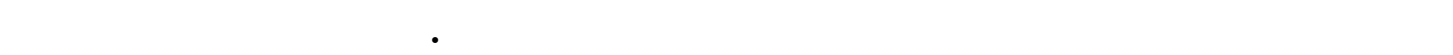

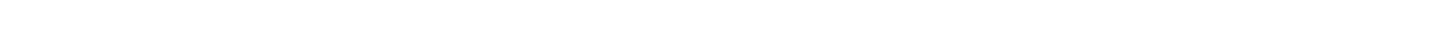
dqg Oxlv Y Ifhlud iru khosixofrp p hqw dqg vxj j hwlrqv1 Ri frxuh/ wkh xvxdofdyhdw dssdhv1 Wkdqnv duh dar gxh w $F$ u wivd Eduf hö ir u khu khos lq surgxflaj wkh j udskv1 Dgguhw iru fruhs rqghqf $\mathrm{h}=\mathrm{F}$ HP I L/ F dvdgr ghoDdvdo 8/ 5; $347 \mathrm{P}$ dgulg/ Vsdlq whol= $.67 \varangle 75<3884 / \mathrm{id}\{=.67 \& 75<4389 / \mathrm{hqp}$ dllo=VhquedqdCfhp $\mathbf{l} \mathbf{N}, 1$
\end{abstract}




\begin{abstract}
In this paper, I first provide a unifying approach to Mean-Variance analysis and Value at Risk, which highlights their similarities and differences. Then I use it to explain how fund managers can take investment decisions within the well-known Mean-Variance allocation framework that satisfy the VaR restrictions imposed on them by regulators. I do so by introducing a new type of line to the usual mean standard deviation diagram, called Iso VaR, which represents all the portfolios that share the same VaR for a fixed probability level. Finally, I analyse the "shadow cost" of a VaR constraint.
\end{abstract}




\section{Introduction}

Mean-Variance analysis and Value at Risk (VaR) are tools routinely used by fund managers all over the world in their portfolio allocation decision-making. Therefore, it is not surprising that vast amounts of academic papers and books have been written on both topics, some of which discuss their relationship (see e.g. Alexander and Baptista (2000), Dowd (1999), Gourieroux and Monfort (2001), or section 14.4 of Jorion (1997)). Nevertheless, there is not yet a simultaneous treatment of the two that is "backward compatible" with the most widely used textbooks on financial investments. In this respect, the purpose of this paper is twofold. First, to offer a simple unifying approach, which highlights the similarities and differences between Mean-Variance analysis and Value at Risk. And second, to explain how fund managers can take informed investment decisions that satisfy the risk-taking restrictions often imposed on them by internal or external regulators without abandoning the well-known mean-variance allocation framework.

I do so by adding a new type of line to the usual mean - standard deviation diagram, which represents all the portfolios that share the same VaR (as a proportion of the available funds) for a fixed probability level. For obvious reasons, I call such lines IsoVaRs. Given some pre-specified risk control parameters, the corresponding IsoVaR effectively constrains the investment opportunity set, which is otherwise characterised by the usual portfolio frontier. Then, I simply have to superimpose the investors' risk preferences to find out the optimal restricted portfolio.

In this respect, this paper is more closely related to Gourieroux and Monfort (2001), who study efficient portfolios for parametric expected utility functions with general risk level constraints, than to Leibowitz and Kogelman (1991) and Alexander and Baptista (2000), who effectively replace mean-variance preferences by mean-VaR preferences. The concept of IsoVaR, though, turns out to be equiv- 
alent to the "shortfall line" introduced by Leibowitz and Kogelman (1991), although their emphasis was on achieving a minimum positive target return over the long run rather than on controlling losses over the short run. ${ }^{1}$

For simplicity of exposition, I make the assumption that a riskless asset is available for unlimited borrowing and lending at a common rate, although one could cope with the alternative assumptions of different rates, no borrowing, or no riskless asset with little additional effort. I also assume throughout that asset returns follow a joint multivariate elliptically symmetric distribution, of which the multivariate normal and the multivariate $t$ are rather special cases. Apart from the obvious fact that many empirical studies indicate that the marginal distribution of asset returns has substantially fatter tails than the normal, and their cross-sectional dependence structure is non-linear, the two main advantages of weakening the standard Gaussianity assumption in this direction are that (i) mean-variance analysis still coincides with expected utility optimisation regardless of the exact form of the preferences, and (ii) the IsoVaRs remain straight lines in mean-standard deviation space.

The rest of the paper is organised as follows. I introduce the three basic building blocks (i.e. portfolio frontiers, indifference curves and IsoVaR lines) in section 2. Then, in section 3 I solve the general mean-variance portfolio allocation with a VaR cap problem, and analyse the "shadow cost" of the constraint. Finally, section 4 contains a brief summary of the results.

\footnotetext{
${ }^{1}$ For a recent, comprehensive analysis of multiperiod investment decisions, see Campbell and Viceira (2001).
} 


\section{The three building blocks}

\subsection{Mean-Variance Portfolio Frontiers}

Consider a world with one riskless asset, and a finite number $N$ of risky assets. Let $R_{s}$ denote the gross return on the safe asset (that is, the total payoff per unit invested), $\mathbf{R}=\left(R_{1}, R_{2}, \ldots, R_{N}\right)^{\prime}$ the vector of gross returns on the $N$ remaining assets, with vector of means and matrix of variances and covariances $\boldsymbol{\nu}$ and $\Sigma$ respectively, which I assume bounded. Let $p=w_{s} R_{s}+w_{1} R_{1}+\ldots+w_{N} R_{N}$ denote the payoffs to a portfolio of the $N+1$ primitive assets with weights given by $w_{s}$ and the vector $\mathbf{w}=\left(w_{1}, w_{2}, \ldots, w_{N}\right)^{\prime}$. Importantly, I assume that there are no transaction costs or other impediments to trade, and in particular, that short-sales are allowed. I also assume that the wealth of any particular investor is such that her individual behaviour does not alter the distribution of returns.

There are at least three characteristics of portfolios in which investors are usually interested: their cost, the expected value of their payoffs, and their variance, given by $C(p)=w_{s}+\mathbf{w}^{\prime} \boldsymbol{\iota}, E(p)=w_{s} R_{s}+\mathbf{w}^{\prime} \boldsymbol{\nu}$ and $V(p)=\mathbf{w}^{\prime} \Sigma \mathbf{w}$ respectively, where $\boldsymbol{\iota}$ is a vector of $N$ ones. Let $\mathcal{P}$ be the set of payoffs from all possible portfolios of the $N+1$ original assets, i.e. the linear span of $\left(R_{s}, \mathbf{R}^{\prime}\right)^{\prime}$. Within this set, several subsets deserve special attention. For instance, it is worth considering all unit cost portfolios $\mathcal{R}=\{p \in \mathcal{P}: C(p)=1\}$, whose payoffs can be directly understood as returns per unit invested; and also all zero cost, or arbitrage portfolios $\mathcal{A}=\{p \in \mathcal{P}: C(p)=0\}$. In this sense, note that any non-arbitrage portfolio can be transformed into a unit-cost portfolio by simply scaling its weights by its cost. Similarly, if $\mathbf{r}=\mathbf{R}-R_{s} \iota$ denotes the vector of returns on the $N$ primitive risky assets in excess of the riskless asset, it is clear that $\mathcal{A}$ coincides with the linear span of $\mathbf{r}$. The main advantage of working with excess returns is that their expected values $\boldsymbol{\mu}=\boldsymbol{\nu}-R_{s} \boldsymbol{\iota}$ directly give us the risk premia of $\mathbf{R}$, without alter- 
ing their covariance structure. On the other hand, one must distinguish between riskless portfolios, $\mathcal{S}=\{p \in \mathcal{P}: V(p)=0\}$ and the rest. In what follows, I shall impose restrictions on the elements of $\mathcal{S}$ so that there are no riskless "arbitrage" opportunities. In particular, I shall assume that $\Sigma$ is regular, so that $\mathcal{S}$ is limited to the linear span of $R_{s}$, and the law of one price holds (i.e. portfolios with the same payoffs have the same cost). I shall also assume that $R_{s}$ is strictly positive (in practice, $R_{s} \geq 1$ for nominal returns).

A simple, yet generally incomplete method of describing the choice set of an agent is in terms of the mean and variance of all the portfolios that she can afford. Let us consider initially the case of an agent who has no wealth whatsoever, which means that she can only choose portfolios in $\mathcal{A}$. In this context, frontier arbitrage portfolios, in the usual mean-variance sense, will be those that solve the program $\min V(p)$ subject to the restrictions $C(p)=0$ and $E(p)=\bar{\mu}$, with $\bar{\mu}$ real. Given that $C(p)=0$ is equivalent to $p=\mathbf{w}^{\prime} \mathbf{r}$, I can re-write this problem as $\min _{\mathbf{w}} \mathbf{w}^{\prime} \Sigma \mathbf{w}$ subject to $\mathbf{w}^{\prime} \boldsymbol{\mu}=\bar{\mu}$. There are two possibilities: (i) $\boldsymbol{\mu}=\mathbf{0}$, when the frontier can only be defined for $\bar{\mu}=0$; or (ii) $\boldsymbol{\mu} \neq \mathbf{0}$, in which case the solution for each $\bar{\mu}$ is

$$
\mathbf{w}^{*}(\bar{\mu})=\bar{\mu}\left(\boldsymbol{\mu}^{\prime} \Sigma^{-1} \boldsymbol{\mu}\right)^{-1} \Sigma^{-1} \boldsymbol{\mu}
$$

As a consequence, the arbitrage portfolio $r_{p}=\left(\boldsymbol{\mu}^{\prime} \Sigma^{-1} \boldsymbol{\mu}\right)^{-1} \boldsymbol{\mu}^{\prime} \Sigma^{-1} \mathbf{r}$ generates the whole zero-cost frontier, in what can be called one-fund spanning. Moreover, given that the variance of the frontier portfolios with mean $\bar{\mu}$ will be $\bar{\mu}^{2}\left(\boldsymbol{\mu}^{\prime} \Sigma^{-1} \boldsymbol{\mu}\right)^{-1}$, in mean-standard deviation space the frontier is a straight line reflected in the origin whose efficient section has slope $\sqrt{\boldsymbol{\mu}^{\prime} \Sigma^{-1} \boldsymbol{\mu}}$. Therefore, this slope fully characterises in mean-variance terms the investment opportunity set of an investor with no wealth, as it implicitly measures the trade-off between risk and return that the available assets allow at the aggregate level.

Traditionally, however, the frontier is usually obtained for unit-cost portfolios, and not for arbitrage portfolios. Nevertheless, given that the payoffs of any port- 
folio in $\mathcal{R}$ can be replicated by means of a unit of the safe asset and a portfolio in $\mathcal{A}$, in mean-standard deviation space, the frontier for $\mathcal{R}$ is simply the frontier for $\mathcal{A}$ shifted upwards in parallel by the amount $R_{s}$. And although now we will have two-fund spanning, for a given safe rate, the slope $\sqrt{\boldsymbol{\mu}^{\prime} \Sigma^{-1} \boldsymbol{\mu}}$ continues to fully characterise the investment opportunity set of an agent with positive wealth.

An alternative graphical interpretation of the same result would be as follows. The trade-off between risk and return of any unit-cost portfolio in $\mathcal{R}$ is usually measured as the ratio of its risk premium to its standard deviation. More formally, if $R_{u} \in \mathcal{R}$, then $s_{u}=\mu_{u} / \sigma_{u}$, where $\mu_{u}=E\left(r_{u}\right), \sigma_{u}^{2}=V\left(r_{u}\right)$, and $r_{u}=R_{u}-R_{s}$. This expression, known as the Sharpe ratio of the portfolio, remains constant for any portfolio whose mean excess return and standard deviation lie along the ray which, starting at the origin, passes through the point $\left(\mu_{u}, \sigma_{u}\right)$. As can be seen in Figure 1, geometrically the Sharpe ratio coincides with the slope of the appropriate ray. As a result, the steeper (flatter) a ray is (i.e. the closer to the $y$ $(x)$ axis), the higher (lower) the corresponding Sharpe ratio.

Then, since $\mu_{p}=1$ and $\sigma_{p}^{2}=\left(\boldsymbol{\mu}^{\prime} \Sigma^{-1} \boldsymbol{\mu}\right)^{-1}$, the slope $s_{p}=\mu_{p} / \sigma_{p}=\sqrt{\boldsymbol{\mu}^{\prime} \Sigma^{-1} \boldsymbol{\mu}}$ will give us the Sharpe ratio of

$$
R_{p}\left(w_{r_{p}}\right)=R_{s}+w_{r_{p}} r_{p}
$$

for any $w_{r_{p}}>0$, which is the highest attainable. Therefore, in mean excess return-standard deviation space, all $R_{p}\left(w_{r_{p}}\right)$ lie on a positively sloped straight line that starts from the origin, as depicted in Figure 1. As the investor moves away from the origin, where she is holding all her wealth in the safe asset, the net total position invested in the riskless asset is steadily decreasing, and eventually becomes zero. Beyond that point, she begins to borrow in the money market to lever up her position in the financial markets. 


\subsection{Mean-Variance Indifference Curves}

The analysis in the previous section shows that any investor endowed with unit wealth who only cares about the mean and variance of her returns will always choose some portfolio $R_{p}\left(w_{r_{p}}\right)$, which exclusively combines $R_{s}$ and $r_{p}$. Nevertheless, the proposed allocation is incomplete, as she still has to determine the degree of leverage of her chosen position, $w_{r_{p}}$.

The traditional solution to such a scale indeterminacy is to parametrise the preferences of the agents in terms of a derived utility function that depends exclusively on the mean and variance of the portfolio returns. Given my assumption that the joint distribution of the primitive asset returns belongs to the elliptical class, ${ }^{2}$ such utility functions are compatible with standard Von-NeumannMorgensten expected utility theory regardless of the specific functional form of the investor's preferences (see Chamberlain (1983) or chapter 4, appendix B in Ingersoll (1987)).

A commonly used specification is:

$$
E\left[R_{u}\right]-\frac{\gamma}{2} V\left[R_{u}\right]=R_{s}+E\left(r_{u}\right)-\frac{\gamma}{2} V\left(r_{u}\right)
$$

where $\gamma$ is a positive risk aversion parameter. The indifference curves associated with such preferences will be parallel straight half-lines with slope $\gamma / 2$ in meanvariance space, and parallel half parabolas in mean-standard deviation space, as depicted in Figure 2. The indifference curves become steeper as the risk aversion coefficient $\gamma$ increases. In particular, they will be almost vertical for an extremely risk averse individual, while they would be flat for a risk neutral agent (i.e. $\gamma=0$ ).

\footnotetext{
${ }^{2}$ The vector of risky asset returns $\mathbf{R}$ will be distributed as an elliptically symmetric multivariate random variable of dimension $N$ if and only if it can be written as an affine transformation of $\varrho_{t} \mathbf{u}_{t}$, where $\mathbf{u}_{t}$ is uniformly distributed on the unit sphere surface in $\mathbb{R}^{N}$, and $\varrho_{t}$ is a non-negative random variable that is independent of $\mathbf{u}_{t}$. When $\varrho_{t}$ is proportional to the square root of an $F$ random variable with $N$ and $\nu$ degrees of freedom, we obtain the multivariate $t$ distribution, which in turn converges to the multivariate normal as $\nu \rightarrow \infty$ (see Fang et al., 1990).
} 
For given risk preferences, though, the utility level of the agent increases as we move up in a northwesterly direction across her indifference curves. ${ }^{3}$

Therefore, it is not surprising that in the absence of any further constraints, her preferred portfolio will be such that the slope of the indifference curve at that point coincides with the maximum Sharpe ratio attainable, as can be seen in Figure 3. For the preferences in (1), in particular, this will happen for $r_{u}=w_{r_{p}}^{M V} r_{p}$, where

$$
w_{r_{p}}^{M V}=\frac{1}{\gamma} \frac{E\left(r_{p}\right)}{V\left(r_{p}\right)}=\frac{1}{\gamma}\left(\boldsymbol{\mu}^{\prime} \Sigma^{-1} \boldsymbol{\mu}\right)=\frac{1}{\gamma} s_{p}^{2}
$$

\section{$2.3 \quad$ IsoVaRs}

Nowadays, however, many institutional investors regularly use risk management procedures based on the ubiquitous Value at Risk to control for the risks associated with their portfolios.

Let $W>0$ denote the initial wealth of an investor. Hence, if $R_{u} \in \mathcal{R}$ denotes the gross return on her chosen portfolio, the random final value of her wealth will be

$$
W R_{u}=W R_{s}+W r_{u}
$$

which contains both a safe component, $W R_{s}$, and a random component, $W r_{u}$. Therefore, the probability that she suffers a reduction in wealth larger than some fixed positive threshold value $V$ will be given by the following expression

$$
\begin{gathered}
P\left[W\left(1-R_{s}\right)-W r_{u} \geq V\right]=P\left(r_{u} \leq 1-R_{s}-V / W\right) \\
=P\left[\left(\frac{r_{u}-\mu_{u}}{\sigma_{u}}\right) \leq \frac{1-R_{s}-V / W-\mu_{u}}{\sigma_{u}}\right]=F\left[\frac{1-R_{s}-V / W-\mu_{u}}{\sigma_{u}}\right]
\end{gathered}
$$

\footnotetext{
${ }^{3}$ The derived utility function in (1) is usually justified by a combination of constant absolute risk aversion and multivariate normality, which are unnecesarily restrictive assumptions for the purposes of this paper. Nevertheless, it helps illustrate the most important features of more general mean-variance preferences.
} 
where $\mu_{u}$ and $\sigma_{u}^{2}$ are the mean and variance of $r_{u}$, and $F($.$) is the cumulative$ distribution function of a zero mean - unit variance random variable within the appropriate elliptical class. ${ }^{4}$ The value of $V$ which makes the above probability equal to some pre-specified value $\alpha(0<\alpha<1 / 2)$ is known as the $100 \alpha \%$ Value at RfJKx7G7zxJx6r9J 


\section{Mean-Variance Portfolio Allocation with a VaR Cap}

As we saw in section 2.1, an investor endowed with unit wealth who only cares about the mean and variance of her returns will always choose some portfolio $R_{p}\left(w_{r_{p}}\right)$, which exclusively combines $R_{s}$ and $r_{p}$. Suppose, however, that because of an internal or external regulation, the investor's overriding concern is that she can only risk losing a fraction $V / W$ or larger of her initial wealth with probability $\alpha$. Then, her effective choice set will be given by the section of the efficient mean-variance frontier between the origin and its intersection with the IsoVaR corresponding to $\alpha$ and $V / W$.

Given that along the efficient frontier $E\left[r_{p}\left(w_{r_{p}}\right)\right]=w_{r_{p}}$ and $V\left[r_{p}\left(w_{r_{p}}\right)\right]=$ $w_{r_{p}} \sigma_{p}$, I can solve for $w_{r_{p}}$ from (2) to find that the intersection point will be:

$$
w_{r_{p}}^{I}=\frac{\left(1-R_{s}-V / W\right)}{1+s_{p} F^{-1}(\alpha)}
$$

as long as the VaR significance level $\alpha$ is such that $1+s_{p} F^{-1}(\alpha)<0$ for $R_{s}-1+$ $V / W \geq 0$. In this respect, note that if $\alpha$ is high enough so that $-F^{-1}(\alpha) \leq 1 / s_{p}$, then $w_{r_{p}}^{I}$ becomes unbounded. The reason is that the overall risk-return tradeoff that the fund manager faces is so good that the portfolio VaR restriction is effectively void.

Therefore, the optimal constrained portfolio for an agent with mean-variance preferences such as (1) will be the minimum of $w_{r_{p}}^{M V}$ and $w_{r_{p}}^{I}$. If the agent is sufficiently risk averse, so that $\gamma$ is high enough, then $w_{r_{p}}^{M V}$ will also solve the restricted problem, while if the agent is less risk averse, then $w_{r_{p}}^{I}$ will be chosen instead. The optimal solution in the latter case is represented in Figure 5.

The previous analysis highlights an important fact that is very often blurred in discussions of the effects of VaR constraints on portfolio allocation. As this figure clearly illustrates, the expected return of the constrained portfolio cannot 
be larger than the expected return of the unconstrained portfolio, because the fund manager must put aside some additional funds in the safe asset to achieve the required VaR when the restriction is binding. Not surprisingly, the risk of the restricted portfolio is also smaller. From the point of view of the fund manager, though, there will be a positive "shadow cost" to a binding risk constraint. Such a cost can be measured in different ways, but perhaps the most useful measure is the reduction in the overall Sharpe ratio that would induce the fund manager to choose an unrestricted portfolio along the same indifference curve as the constrained one. ${ }^{5}$ Graphically, this is given by the differences in slope between the actual and the "shadow" efficient frontiers in Figure 5.

\section{Summary}

In this paper I provide a unifying approach to Mean-Variance analysis and Value at Risk, which can be used to carry out informed investment decisions within the well-known mean-variance allocation framework that satisfy the Value at Risk restrictions often imposed on fund managers by internal or external regulators.

This is achieved by introducing a new type of line to the usual mean - standard deviation diagram, which I call Iso VaR, which represents all the portfolios that share the same Value at Risk (as a proportion of the available funds) for a fixed probability level $\alpha$. Given some pre-specified risk control parameters, I show that the restricted portfolio allocation decision can be solved by simply superimposing the investors' mean-variance risk preferences on the section of the efficient frontier that is delimited by the appropriate Iso VaR.

Importantly, I show that from the point of view of the fund manager, there will be a "shadow cost" to a binding VaR constraint. This cost simply reflects the

\footnotetext{
${ }^{5}$ For instance, for the mean-variance preferences in (1), the "shadow" Share ratio, $\mathrm{s}_{p^{*}}$ say, would be given by the formula $\mathrm{s}_{p^{*}}=\sqrt{2 \gamma w_{r_{p}}^{I}\left[1-\left(\gamma w_{r_{p}}^{I}\right) /\left(2 s_{p}^{2}\right)\right]}$.
} 
fact that the VaR restriction is imposed on the fund manager by an internal or external regulator, whose risk attitudes and objectives are different from the risk attitudes and objectives of the manager. 


\section{References}

Alexander, G.J. and A.M. Baptista. 2000. "Economic Implications of Using a Mean-VaR Model for Portfolio Selection: A Comparison with Mean-Variance Analysis", Working Paper, University of Minnesota (December).

Campbell, J.Y. and L.M. Viceira. 2001. Strategic Asset Allocation: Portfolio Choice for Long-Term Investors, forthcoming in Oxford University Press.

Chamberlain, G. 1983. "A Characterization of the Distributions that Imply Mean-Variance Utility Functions", Journal of Economic Theory vol. 29, no. 1 (January): 185-201.

Dowd, K. (1999): "A Value at Risk Approach to Risk-Return Analysis", Journal of Portfolio Management, vol. 25, no. 4 (Summer): 60-67.

Fang, K.-T., S. Kotz, S. and K.-W. Ng. 1990. Symmetric Multivariate and Related Distributions, Chapman and Hall.

Gourieroux, C. and A. Monfort. 2001. "The Econometrics of Efficient Portfolios", Working Paper, CREST-INSEE (February).

Ingersoll, J.E. (1987): Theory of Financial Decision Making, Rowman and Littlefield, Maryland.

Jorion, P. (1997): Value at Risk, McGraw-Hill, New York.

Leibowitz, M.L. and S. Kogelman, S. 1991. "Asset Allocation under Shortfall Constraints", Journal of Portfolio Management vol. 17, no. 2 (Winter): 18-23. 
Figure 1

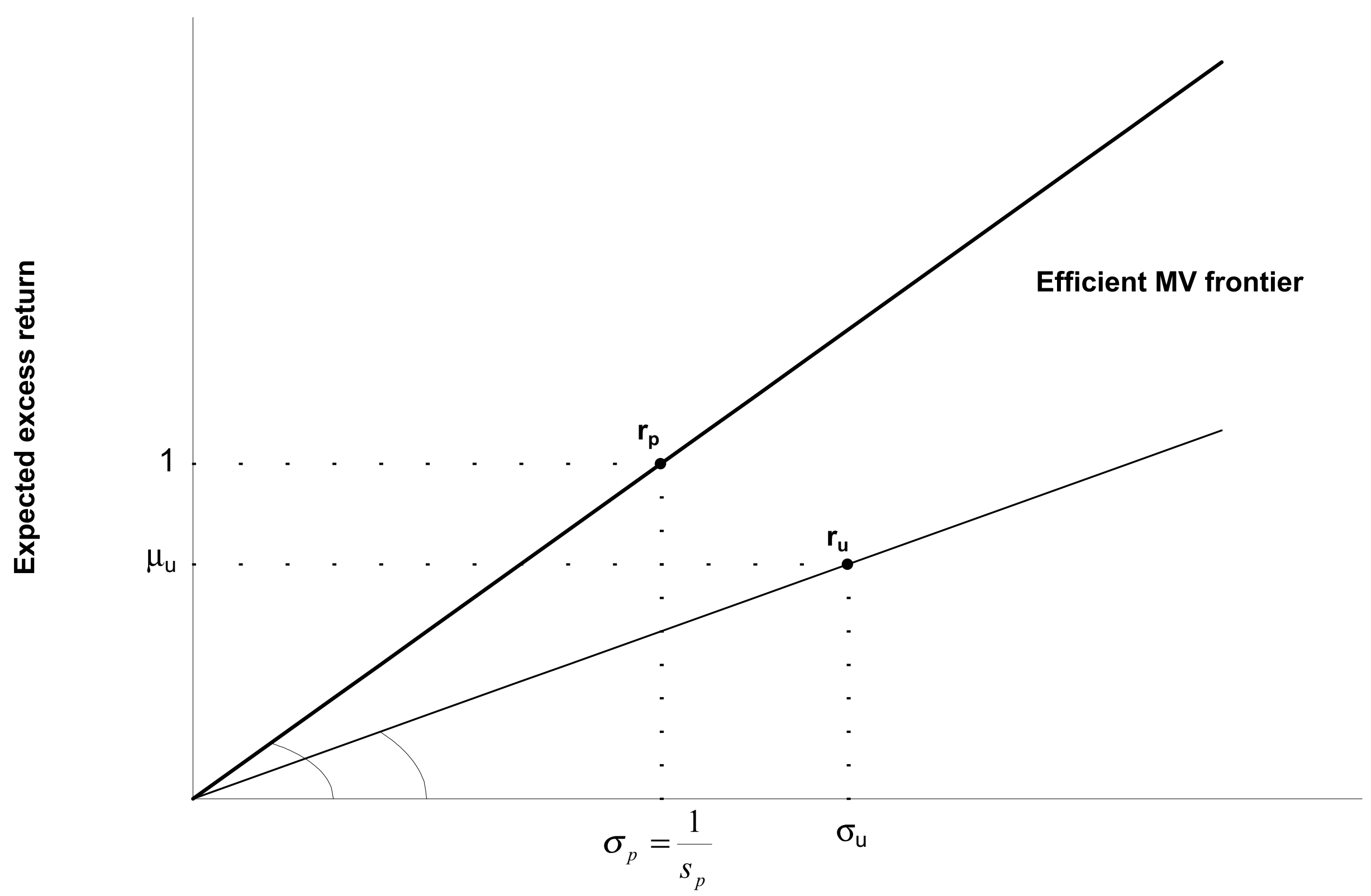

Standard deviation 
Figure 2

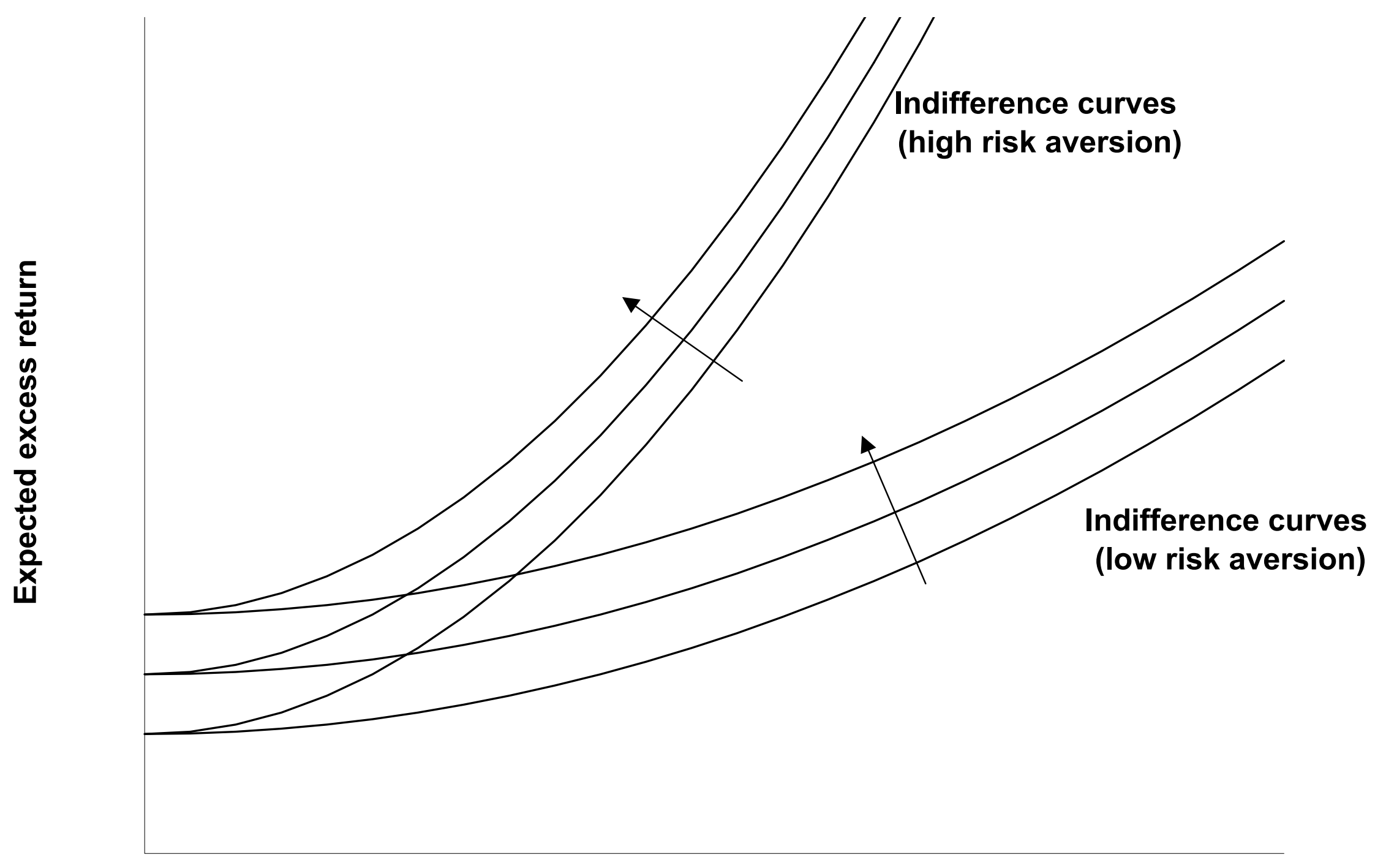

Standard deviation 
Figure 3

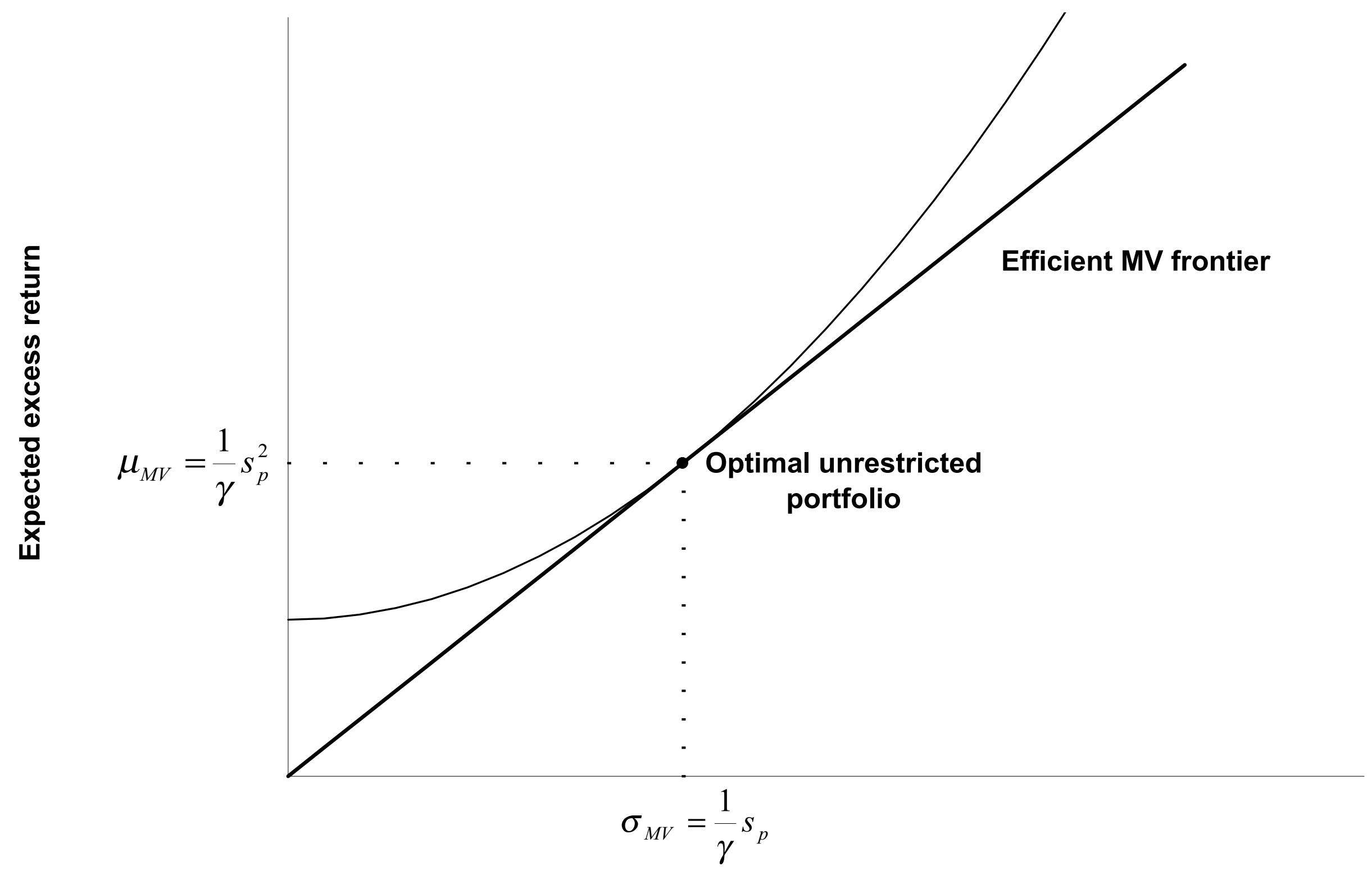

Standard deviation 
Figure 4

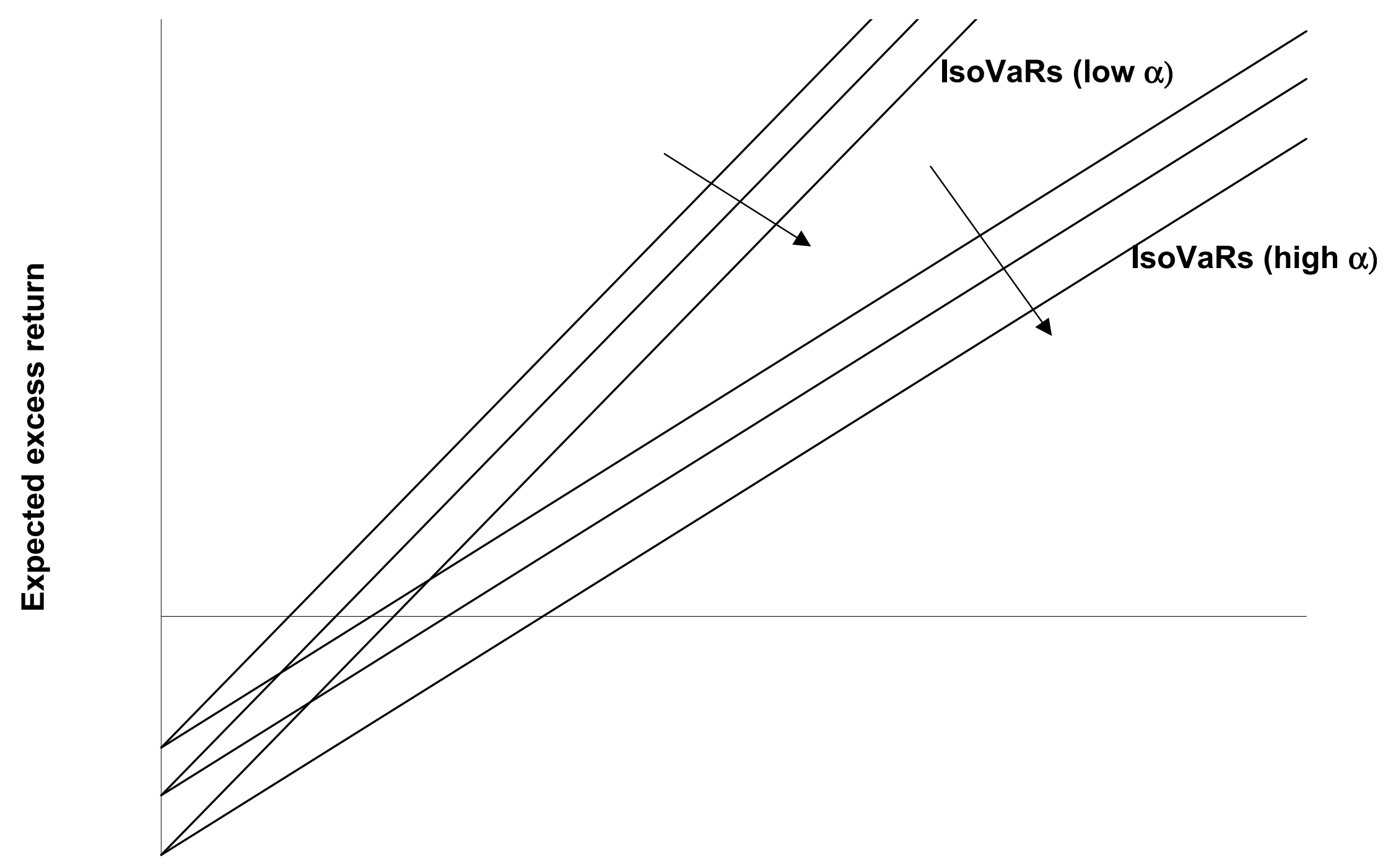

Standard deviation 


\section{Figure 5}

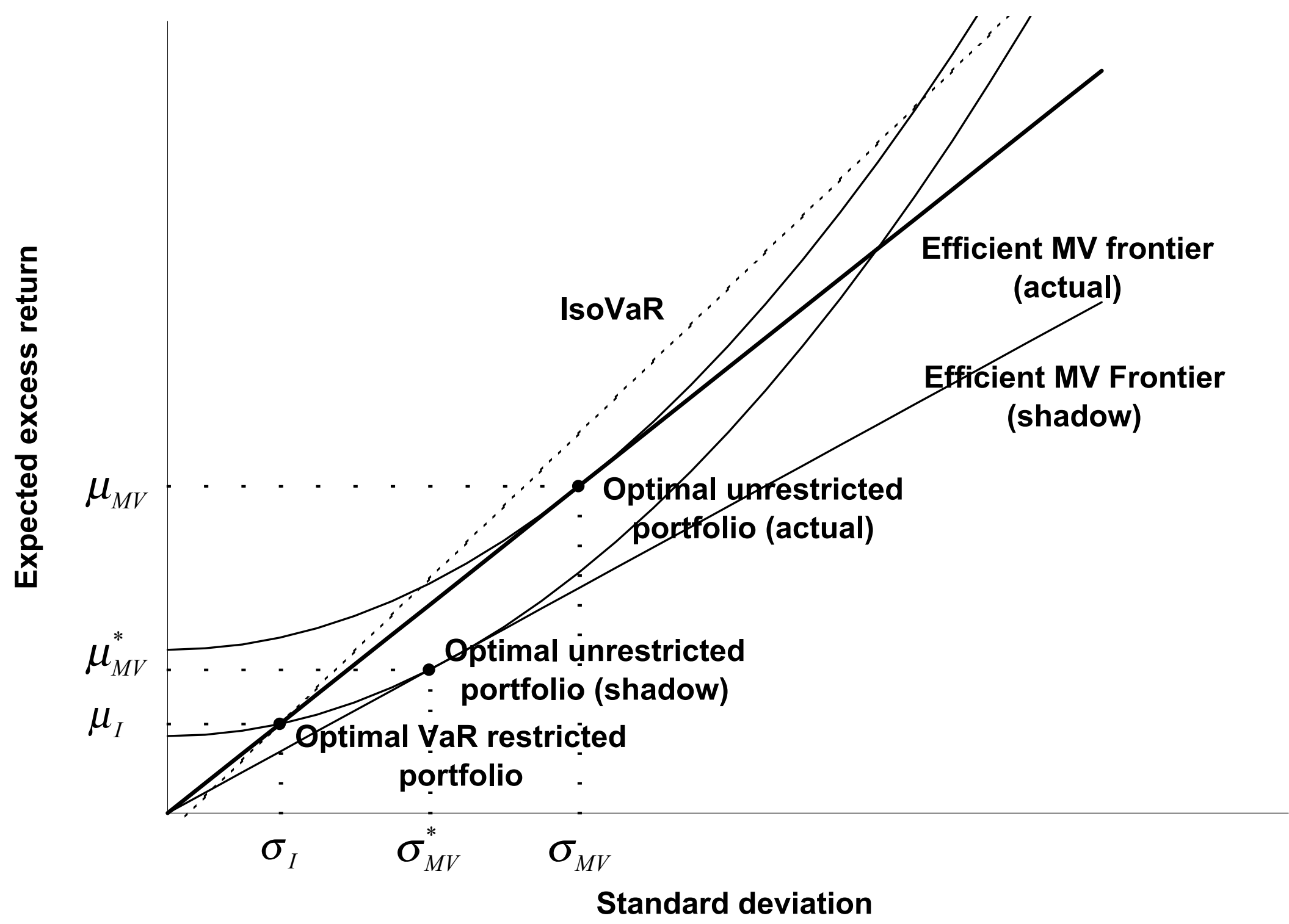

